\title{
LOS PRESUPUESTOS PSICOLÓGICOS EN POLÍTICA UNA REVISIÓN INTRODUCTORIA DE LA LITERATURA
}

\author{
Alejandra Salinas \\ Universidad Católica Argentina/Eseade
}

\begin{abstract}
Resumen: El análisis de las disposiciones psicológicas de los actores políticos, sus implicancias institucionales y su impacto en las políticas públicas ha recibido creciente atención académica en las últimas décadas. Creemos que el estado del arte amerita una revisión introductoria que oriente al lector respecto de las principales posturas teóricas sobre el tema. A tal fin, este trabajo resume, clasifica y compara teorías de autores clásicos como de otros más recientes, e incorpora contribuciones de diversas disciplinas.
\end{abstract}

Palabras clave: psicología, política, racionalidad, ignorancia, emociones.

Recibido: enero 2011; aceptado: octubre 2011.

Alejandra Salinas. Licenciada en Ciencias Políticas y Doctora en Sociología, Universidad Católica Argentina (UCA). Profesora de Teoría Política y Social en UCA y Eseade (salinas@eseade.edu.ar).

* Versión adaptada del trabajo elaborado gracias a la Beca Federico Zorraquín/Eseade, presentado en el Congreso Nacional de Ciencia Política (Santa Fe, agosto de 2009) y en la Universidad de San Andrés (noviembre de 2009). Agradezco los comentarios allí recibidos y las sugerencias de los referees de Estudios Públicos. 


\title{
PSYCHOLOGICAL ASSUMPTIONS IN POLITICS. AN INTRODUCTORY REVIEW OF THE LITERATURE
}

\begin{abstract}
The analysis of the psychological dispositions of political actors, their institutional implications and impact on public policy has received increasing academic attention in recent decades. We believe that the state of the art merits an introductory review to guide the reader on the major theoretical positions on the issue. To this end, this paper summarizes, classifies and compares theories of classical and contemporary authors, and includes contributions from various disciplines.
\end{abstract}

Keywords: psychology, politics, rationality, ignorance, emotions.

Received: January 2011; accepted: October 2011.

\section{Introducción}

$\mathbb{Q}_{1}$ estudio de las disposiciones psicológicas de los actores políticos y las implicancias que ellas tienen para la organización y el funcionamiento institucional ha sido ampliamente ilustrado en el pensamiento moderno. Así, Hobbes explicaba el contrato social a partir del miedo provocado por la agresión mutua; Adam Smith consideraba el dominio de sí mismo como una de las cualidades sociales más útiles en una sociedad libre; Kant detectaba la pereza y la falta de coraje como el mayor obstáculo para cumplir con el motto de "pensar por uno mismo" y alcanzar así la autonomía; Locke advertía que las personas suelen ser "lentas y perezosas" para cambiar sus inclinaciones y proclives a soportar una "larga cadena de abusos", y Tocqueville observaba que, debido a la moderna división del trabajo, los votantes juzgaban "apresuradamente", prefiriendo "el más saliente de los objetos"1.

A partir de sus observaciones, estos autores imaginaron arreglos institucionales que pudieran atenuar, entre otras cosas, los problemas asociados con las limitaciones cognitivas y emocionales de los individuos. Salvo excepciones, sus escritos han sido accesibles al lector

${ }^{1}$ Hobbes, Leviathan, Cap. XIII; Smith, The Theory of Moral Sentiments, Parte VI: Conclusión; Kant, "What is Enlightenment?"; Locke, Second Treatise, Cap. XIX: Sec. 223-225; Tocqueville, La democracia en América, Vol. II: Cap. 5. 
interesado en comprender los fenómenos sociales y políticos y las complejas motivaciones y tendencias humanas subyacentes a ellos.

De un tiempo a esta parte, los aportes teóricos cobraron un giro más técnico y especializado, acompañado de la multiplicación y variedad disciplinaria de textos, lo que dificulta una lectura general e introductoria sobre el tema. Por ello, este trabajo busca ofrecer una clasificación y comparación general de distintas teorías y disciplinas en torno a los presupuestos psicológicos en política, incluyendo un esbozo general de sus implicancias institucionales y de su impacto en las políticas públicas.

Cabe aclarar que la expresión "disposiciones psicológicas" alude aquí, en un sentido amplio, a dos dimensiones de los procesos mentales de las personas: la dimensión racional o cognitiva (proceso de pensamiento lógico / uso de las facultades racionales para obtener y procesar información sobre la realidad / elección de los medios para la consecución óptima de un $\mathrm{fin}^{2}$ ) y la dimensión emocional o sentimental (sensaciones y percepciones frente a ciertos estímulos / estados de ánimo / motivaciones para obrar en base a sentimientos) ${ }^{3}$. Ambas dimensiones son aplicadas al análisis de las opiniones, decisiones y conductas de los actores políticos, es decir, de los ciudadanos (en cuanto votantes), los expertos o técnicos, los funcionarios de gobierno, los dirigentes y demás impulsores de políticas públicas. Por razones de espacio, deberé centrar mi trabajo en las teorías sobre las cualidades cognitivas en mayor medida que en los sentimientos, y entre los actores políticos, en los ciudadanos o votantes en mayor medida que en los expertos o técnicos, dirigentes y funcionarios.

${ }^{2}$ Esta breve definición busca ilustrar las distintas facetas de la racionalidad que conviven en la mente humana: la instrumental (analizar la utilidad de medios con relación a fines), la axiológica y normativa (razonar en base a creencias y valores considerados verdaderos o buenos), y la gnoseológica (conocer la realidad y procesar bien la información).

${ }^{3}$ Antonio Damasio distingue entre las emociones como respuesta frente a un estímulo y la percepción de esa respuesta (2005:270). Andrew Gluck amplía el concepto para incluir sensaciones y percepciones relativas a particulares estados psíquicos y espirituales —además de físicos-, y a los estados de ánimo (feeling-states) (2007:112-113). Por su parte, Robert Frank (1988) y Viktor Vanberg (2006) defienden la función motivadora de las emociones para adoptar cursos de acción considerados óptimos desde un punto de vista evolutivo. 
El análisis de las cualidades y procesos cognitivos ha recibido intensa y creciente atención académica de diversas disciplinas. Entre los problemas advertidos por quienes plantean la "racionalidad limitada" y las "disonancias cognitivas" de las personas en su calidad de tomadores de decisiones (calidad medida a partir de observaciones y estudios experimentales) figuran la tendencia de los votantes a juzgar intuitivamente (Kahneman, 2002), su dificultad para procesar diferentes piezas de información de modo simultáneo (Simon, 1985) o su dificultad para comprender los aspectos ideológicos al momento de votar (Converse, 1964). Por otro lado, entre las falencias cognitivas de los funcionarios y expertos, se ha señalado la pretensión de conocer aquellas circunstancias que sólo los individuos pueden conocen actuando en un orden de mercado (Hayek, 1978), la simplificación que hacen de las teorías (Rizzo y Whitman, 2008), la predisposición a la "racionalidad insincera" de los políticos (Pincione y Tesón, 2006), y la preeminencia de la ideología o el dogmatismo por sobre la constatación empírica (Friedman, 2005), entre otras. Común a todos los actores políticos es la tendencia a ignorar el hecho de que las consecuencias o resultados suelen no seguirse de las intenciones invocadas o los fines buscados individual o colectivamente (Friedman, 2005).

Entre las implicancias institucionales de los problemas antes mencionados se ha sostenido, por ejemplo, que dada la limitada capacidad cognitiva de los actores políticos la democracia sería una institución subóptima respecto de los mercados (Rizzo y Whitman, 2008; Caplan, 2007; Pincione y Tesón, 2006; Tebble, 2003). Otros recalcan la necesidad de reforzar las prácticas deliberativas en los espacios públicos para que las personas puedan formar o mejor conocer sus preferencias (Rawls, 1997; Nino, 1996), y/o defienden medidas "paternalistas" que guíen a las personas a tomar decisiones en la dirección racionalmente óptima respecto de un objetivo dado (Thaler y Sustein, 2003). Se encuentran también quienes impulsan la necesidad de "filtros institucionales" para corregir las limitaciones cognitivas de los votantes (Brennan y Lomasky, 1993; Hardin, 2000), mientras otros promueven la limitación del poder político como una forma de atenuar las consecuencias de los problemas cognitivos tanto de los votantes (Somin, 1998; Brennan y Buchanan, 2000) como de los funcionarios y técnicos (Gaus, 2008; Weyland, 2006). 
Por otro lado, en términos de la influencia de los sentimientos, algunos autores han analizado el modo en que éstos se manifiestan en la vida política. Una preocupación común ha sido abordar el problema de los condicionamientos psicológicos a la libertad individual, asociándolos por ejemplo con los "miedos y complejos, ignorancia, error, prejuicios, ilusiones, fantasías, compulsiones" (Berlin, 1983:306). En líneas similares, el miedo a la incertidumbre y el sentimiento de inseguridad, se ha sostenido, impulsarían a las personas a exigir al gobierno una posición material mínima asegurada contra todo riesgo. Esta demanda habría provocado un Estado sobre-extendido, con problemas de eficiencia, burocracia y falta de transparencia (Buchanan, 2005; Hayek, 1978).

Dado que la literatura sobre el tema de este trabajo es muy variada, es difícil no incurrir en una selección relativamente arbitraria y simple de los argumentos ofrecidos en este campo. Al respecto he escogido aquellos que, creo, ilustran debates clásicos tanto como actuales, y cuyos presupuestos psicológicos tienen importantes implicancias institucionales y para las políticas públicas. Por otro lado, cabe aclarar que en las teorías aquí presentadas conviven los enfoques predictivo, descriptivo, normativo y evaluativo: el primero busca anticipar ciertos cursos de acción y sus consecuencias a partir de presupuestos metodológicos; el descriptivo caracteriza cómo la gente de hecho razona o siente; el normativo se propone indicar una regla, proceso o principio ideal a alcanzar, y el evaluativo busca determinar en qué medida los conceptos y conductas concuerdan con esos ideales.

A partir de esta distinción analítica, y a título ilustrativo, mencionaré que los psicólogos cognitivos suelen ofrecer un enfoque descriptivo que, a partir de estudios experimentales, busca anticipar ciertas falencias o "disonancias cognitivas" de los actores individuales (Kahneman y Tversky, 1984). Otros emplean una mirada descriptiva para cuestionar el alcance de los estudios que miden la racionalidad del votante en base a complicados cuestionarios elaborados por expertos (Lupia, 2006). En tanto, las teorías normativas asumen a priori ciertos presupuestos psicológicos para construir su edificio teórico y efectuar recomendaciones, institucionales y otras (Brennan y Hamlin, 2000; Habermas, 1999, Rawls, 1997). Mientras que la mirada descriptiva observa conductas y opiniones para indagar cuáles son los problemas cognitivos y cómo se manifiestan en la realidad bajo estudio, la mirada normativa se pregunta, por ejemplo, qué instituciones o medidas deben 
adoptarse para asegurar mejor los resultados consistentes con ciertos valores. Finalmente, la mirada evaluativa se detiene a analizar si determinados presupuestos psicológicos posibilitan o no el cumplimiento de los ideales normativos. Así, como veremos, Anthony Downs (1957) elabora el presupuesto de la ignorancia racional y evalúa bajo qué condiciones el ideal de la igualdad democrática se ve afectado por ella, mientras que James Buchanan (2005) examina el sentimiento del miedo a la incertidumbre para evaluar su impacto respecto del ideal de libertad individual.

Hechas estas aclaraciones, nuestro recorrido será el siguiente: en la sección 1 se clasifican los argumentos sobre la capacidad cognitiva de los actores políticos, es decir, el grado en que éstos poseen o pueden poseer el conocimiento suficiente para tomar decisiones racionales, y se presentan algunas implicancias institucionales que se derivan de ellos. En la sección 2 analizo algunas consecuencias para las políticas públicas a la luz de esos argumentos. La sección 3 trata sobre las emociones en política y sus implicancias para el diseño y funcionamiento de ciertos programas e instituciones políticas. Concluyo el trabajo con un resumen de sus principales ideas.

\section{Argumentos sobre la racionalidad en política}

El desafío que presenta el aspecto cognitivo para el diseño y funcionamiento de las instituciones políticas queda expresado en las siguientes preguntas: ¿son los votantes racionalmente capaces de adquirir información correcta, y de actuar políticamente en forma coherente y reflexiva? ¿En qué medida poseen falencias cognitivas (¿cuáles serían?) que dificultan esa tarea? ¿Acaso los gobernantes y los técnicos exhiben falencias similares o distintas? ¿Qué problemas se presentan a partir de las limitaciones respectivas?

A continuación distingo cinco grupos de posturas sobre la racionalidad en política, bajo los rótulos de: a) ignorancia inducida, b) elección racional, c) racionalidad deliberativa, d) racionalidad limitada, y e) ignorancia inevitable. Las primeras dos corresponden a teorías que son en cierto modo fundacionales, ya que a partir de ellas se ha desarrollado una extensa literatura sobre el tema. La primera presenta una mirada más descriptiva, y la segunda es más predictiva. Las otras cuatro posturas están ordenadas por el mayor al menor grado de racionalidad 
que reconoce cada una. Mientras c) y e) tienden a ser más normativas, d) enfatiza los aspectos empíricos.

En base a esta somera distinción, señalaré las principales contribuciones de estos planteamientos y algunas similitudes y diferencias entre ellos. Brevemente y en líneas generales, anticiparé que a) y d) detectan serios problemas cognitivos en los votantes, pero asumen que los políticos y expertos poseen cualidades cognitivas suficientes para prevenir o corregir los efectos políticos derivados de esos problemas. En contraste, b) y c) defienden un presupuesto más exigente de racionalidad, si bien difieren en sus enfoques e implicancias: la "elección racional" asume un cálculo individual de costo-beneficio que conduciría a una débil participación política por parte de los votantes, mientras que los "deliberativos" asumen la capacidad racional de éstos para deliberar sobre los asuntos públicos. Por último, la "ignorancia inevitable" mantiene el supuesto cognitivo más débil y lo aplica tanto a los votantes como a los políticos, funcionarios y expertos.

\section{a) Ignorancia inducida}

Si bien no lo desarrolló en profundidad, a mediados de los años 40 Joseph Schumpeter planteó argumentos que están todavía vigentes sobre la racionalidad del ciudadano promedio, a quien veía guiado por promesas electorales de corto plazo e inserto en un sistema donde las responsabilidades inmediatas son difusas. Según este autor, el votante escapa a la "influencia racionalizadora de la experiencia y la responsabilidad personal" y se sitúa en un plano de "inferioridad mental". De ahí su "ignorancia y falta de juicio" en asuntos políticos complejos, incluso de la gente instruida, pese a la abundancia de información. Esto tendría dos consecuencias negativas: dejarse llevar por asociaciones fáciles e impulsos y prejuicios, y así favorecer a los grupos que moldean las opiniones políticas (Schumpeter, 1947:302-304).

La teoría de Schumpeter fue catalogada como una que asume la "irracionalidad" de los votantes (Prisching, 1995; Wohlgemuth, 2007). En mi opinión, si bien el autor usa el término "irracional", lo aplica para describir los efectos del sistema institucional sobre la conducta particular del votante, antes que para describir las cualidades cognitivas de éste. De hecho, el autor reconoce que "frecuentemente se exagera la 
irracionalidad individual", porque se confunde la racionalidad de pensamiento con la racionalidad de acción, que puede existir sin "ninguna deliberación consciente". Schumpeter cree, además, que las personas suelen reaccionar de forma rápida y racional frente a beneficios pecuniarios (pagos directos, tarifas protectoras, etc.), si bien sostiene que es una racionalidad de corto plazo, que puede equivocarse respecto de esos beneficios a largo plazo (ibídem, 300,304).

Bajo esta óptica, es plausible pensar que en Schumpeter la "ignorancia y la falta de juicio" del votante no proceden de anomalías cognitivas propiamente dichas sino de un sistema que exige a los votantes tomar decisiones sin el correlato de enfrentar la responsabilidad de las consecuencias por las decisiones tomadas. En un párrafo muy citado, el autor observa que el ciudadano "emplea un esfuerzo menos disciplinado para dominar un problema político que el que emplea en una partida de bridge" donde las tareas y las reglas son claras y la responsabilidad por los resultados es atribuida a cada individuo (ibídem, 303). Por lo tanto, sin el incentivo que procede de enfrentar responsabilidades inmediatas, el autor concluye que esa ignorancia persistirá, a pesar de la disponibilidad de información y de la capacidad para interpretarla. Es decir, las características del sistema introducen incentivos para que el votante no despliegue su capacidad cognitiva potencial para adquirir y evaluar la información política.

El problema del comportamiento electoral así entendido estaría fomentado además por la actitud de los políticos que se ven tentados a manipular la ignorancia con fines políticos. En contraste con el votante “infantil”, el político schumpeteriano se presenta como un líder con capacidad suficiente para moldear la opinión de los votantes, y que compite con otros para hacerlo. Así, la democracia sería un proceso de competencia electoral entre políticos que "crean" las preferencias del votante, frecuentemente escondiendo información o mintiendo a favor de su propio interés (ibídem, 305-306) ${ }^{4}$. Sin embargo, los líderes sólo parecieran utilizar estos artilugios principalmente en el momento electoral para acceder al poder, puesto que una vez en el gobierno Schumpeter cree en la posibilidad de que existan líderes con cualida-

${ }^{4}$ En líneas similares véase Russell Hardin (2000:121) para la noción del político schumpeteriano como experto que puede juzgar mejor lo que sirve a los intereses del electorado. 
des "suficientemente elevadas" que aseguren el éxito de la democracia (ibídem, 335-337) .

Al margen de esas cualidades, las implicancias institucionales del modelo cognitivo de Schumpeter se traducen en la confianza en que pueda existir un grupo de funcionarios, esto es, una burocracia fuerte, eficiente, e independiente del poder político, con la suficiente capacidad como para instrumentar un gobierno eficaz (ibídem, Cap. 23).

Una teoría cognitiva similar a la de Schumpeter es la de James M. Buchanan, para quien la incertidumbre sobre los resultados electorales y la pérdida del sentido de la responsabilidad personal en la toma de decisiones políticas limitan el presupuesto de racionalidad individual (Buchanan y Tullock, 1999:37-39). En tanto el presupuesto de racionalidad instrumental basado en el análisis costo-beneficio permite predecir el comportamiento individual con éxito en el campo económico, en política los votantes tenderían a guiarse por cuestiones ideológicas o por consideraciones meramente expresivas o simbólicas (Buchanan y Tullock, 1999:12). Así, la elección del votante sería de tipo "litúrgica" (análoga a la de alentar a un equipo deportivo), por lo que no se puede esperar que se seleccionen las opciones que reflejen los intereses racionalmente evaluados (Brennan y Buchanan, 2000:160-166).

También como Schumpeter, para Buchanan la limitación cognitiva de los votantes se debería al marco institucional, ya que tanto el homo economicus como el homo politicus responderían a la estructura de incentivos institucionales en que se insertan: mientras las preferencias del primero tienen correlato directo con los resultados esperados de su actividad de intercambio, las preferencias del segundo se agregan mediante reglas de decisión mayoritaria que no reflejan esas preferencias en los resultados obtenidos. A ello se suma la complejidad de las cuestiones económicas y políticas, frente a la cual ni siquiera los expertos concuerdan sobre las consecuencias de las medidas de gobierno, y frecuentemente se equivocan (Buchanan y Wagner (2000): 132-133) ${ }^{6}$.

\footnotetext{
${ }^{5}$ Schumpeter señala la necesidad del autocontrol de los votantes y los parlamentarios para resistir los "ofrecimientos de pillos y desequilibrados" y la "tentación de derribar u obstaculizar al gobierno cada vez que tienen ocasión de hacerlo" (ibídem, 340). En última instancia, las conductas de votantes y políticos en la democracia son analizadas a la luz de sus cualidades morales.

6 Véase también Douglas North (1990) para la necesidad de insertar el debate sobre la racionalidad dentro de un marco institucional que disminuya los costos de transacción y facilite el manejo de la información en la toma de decisiones políticas.
} 
A la luz de este diagnóstico, y en contraste con el burócrata eficiente de Schumpeter, desde el punto de vista institucional Buchanan ha propuesto optar por los mercados y la descentralización política para proveer los bienes buscados por los ciudadanos, antes que por procesos electorales mayoritarios a gran escala (Brennan y Buchanan, 2000:168).

\section{b) Elección racional}

La teoría de la elección racional asume los presupuestos de información completa y racionalidad instrumental de todos los actores políticos, quienes usarían un análisis utilitario de costo-beneficio para evaluar, y consecuentemente elegir, aquella opción más beneficiosa o menos costosa en términos individuales ${ }^{7}$.

El modelo de la elección racional ha sostenido que los votantes tienden a no informarse adecuadamente sobre los temas o candidatos políticos, es decir, eligen permanecer "racionalmente ignorantes". Esta elección obedecería a los incentivos introducidos por las siguientes características del sistema representativo actual: la incertidumbre del resultado electoral, el bajo peso relativo del voto individual en determinar el resultado de la votación, el costo de obtener información adecuada con la cual fundamentar el voto, y el carácter indivisible de los bienes públicos que imposibilita desagregar las opciones votadas presentándolas en vez como un bloque. En este contexto de incertidumbre y de altos costos, que el votante invierta en información sería "irracional” (Downs, 1957).

La ignorancia racional se reflejaría por ejemplo en el desconocimiento de los votantes de las plataformas partidarias y de las instituciones y funciones básicas del gobierno, así como en la incapacidad del votante medio para analizar múltiples asuntos mediante un marco analítico integrado (Somin, 1998: 416-418). La situación se vería exacerbada por el crecimiento en el tamaño y el alcance del sector público, que dificulta el conocimiento de los temas y candidatos políticos, así como por los incentivos introducidos por un Estado con poderes redistributivos, que dispersa los costos de la redistribución y concentra sus beneficios en algunos sectores (Somin, 1998:433-435).

${ }^{7}$ La lógica de la elección racional aplicada al caso de las decisiones políticas y grupales fue inicialmente analizada in extenso por Anthony Downs (1957) y Mancur Olson (1971), respectivamente. 
Profundizando el argumento sobre la ignorancia racional, recientemente Guido Pincione y Fernando Tesón han sugerido que los votantes no sólo eligen ignorar datos e información política, sino también ignoran las teorías respecto de las políticas públicas que mejor asegurarían los resultados preferidos por ellos. Así, los autores hablan de "fallas del discurso" para aludir a un fenómeno social caracterizado por la combinación de tres aspectos mutuamente reforzados: la ignorancia racional de la mayoría de los votantes, el aprovechamiento que los políticos hacen de ella (posturing), y el moderno Estado redistribuidor (Pincione y Tesón, 2006: 5,16-18). En directa crítica a las teorías deliberativas que veremos más adelante, para estos autores en un escenario así retratado los incentivos de los actores políticos tenderán a alejarlos de la "verdad", al contrario de lo que postulan quienes confían en el debate público como método racionalmente óptimo para llegar a ella.

Una de las implicancias institucionales del modelo de la elección racional es que la disparidad de información se traduce en desigualdad política, es decir, que las decisiones democráticas no reflejan la igualdad de preferencias que alegan representar (Downs, 1957:257). Así, los grupos de presión y las minorías organizadas estarían dispuestos a hacer frente a los costos de obtener información para influir en el gobierno, lo que favorecería a estos grupos a expensas de la ciudadanía en general (Downs, 1957:254-256). Es decir, una de las peores consecuencias de este escenario cognitivo sería la "captura" de las políticas públicas por parte de grupos especiales que buscan aumentar sus ingresos o rentas a través de la acción pública (Pincione y Tesón, 2006:5).

Bajo esta luz, se ha recalcado la necesidad de imaginar nuevas propuestas donde las personas tengan los incentivos adecuados para tomar decisiones conducentes a alcanzar sus objetivos (Pincione y Tesón, 2006: 228,231-232). Una de las recomendaciones normativas en este sentido es adoptar un sistema de gobierno con funciones y alcances más limitados, que atenúen los problemas cognitivos agudizados en presencia de un poder político monopólico y demasiado extendido (Somin, 1998:446).

La literatura sobre el tema de la ignorancia racional es extensa y no pretendo revisarla exhaustivamente sino sólo para contrastar su idea principal con las otras posturas aquí analizadas. Al igual que Schumpeter, la escuela de la elección racional reconoce en el votante la existencia de una capacidad cognitiva fuerte, que en el análisis de Schumpeter 
es "bloqueada" por un sistema que no le exige utilizarla, y en el de Downs es "utilizada" para evadir el elevado esfuerzo de adquirir información, ya que ello no le depara beneficios ciertos o directos. En ambos casos, el votante permanece desinformado, lo que facilitaría a los políticos manipular la (escasa) información de modo de maximizar los votos a obtener y, una vez en sus cargos, intentar maximizar el poder que les es conferido.

Las críticas a este modelo son numerosas. Brevemente apuntaremos que la imagen del individuo efectuando un cálculo basado en el supuesto de racionalidad instrumental ha recibido la crítica de quienes señalan su carácter restringido e incapaz de explicar otros tipos de racionalidad, tal como la racionalidad axiológica observada por Max Weber (Boudon, 2003), o como el compromiso individual para obedecer reglas morales antes que la persecución del beneficio material (Vanberg, 2006). También se ha resaltado, con Buchanan, la fuerza del voto "simbólico" guiado por razones no utilitarias y por ideas relacionadas con la defensa de valores como la igualdad, la justicia y la verdad (Nozick, 1995:49-60). Adicionalmente, se ha señalado que nadie sabe exactamente qué es lo que tendría que calcular para proceder a invertir en obtener información política (Wohlgemuth, 2007:11-13), o para evaluar todos los efectos de una legislación dada sobre el bienestar individual (North, 1990:360).

Finalmente, una de las críticas más difundidas actualmente es la de Kahneman y Tversky (1984), quienes a partir de observaciones en el campo económico cuestionan el presupuesto de la elección racional y defienden un modelo de racionalidad limitada, basado en la preeminencia de ciertos condicionamientos psicológicos (aversión al riesgo, influencia del encuadre, etc.) por sobre el cálculo racional utilitario. Volveré sobre esta última noción en la próxima sección.

\section{c) Racionalidad limitada}

Desde una perspectiva descriptiva, algunos teóricos de la racionalidad limitada asocian los problemas cognitivos principalmente con la conducta y opiniones de los votantes, quienes, en el mejor de los casos, son racionales respecto de aspectos de la realidad política muy reducidos y desconectados (Simon, 1985:302). Para otros, la ignorancia del votante proviene del presupuesto de que están "mal equipados para 
aislar la información correcta" (Brennan y Lomasky, 1993:209). Bajo esta óptica, la mayor parte de las personas no estaría capacitada para procesar información política, deliberar sobre propuestas alternativas o evaluar las implicancias y resultados de las políticas públicas. Sólo estarían capacitadas para seleccionar candidatos y recibir información de los partidos políticos, los que son fáciles de clasificar (Brennan y Lomasky, 1993:217). En este sentido, las teorías de racionalidad limitada se distinguen de las teorías de la ignorancia inducida y de la elección racional en que para las primeras existen anomalías cognitivas propias de la mayoría de las personas que no pueden revertirse, mientras que para las segundas son las instituciones las que condicionan la habilidad cognitiva del individuo promedio e impiden alcanzar los beneficios generales que se derivarían del uso de esa habilidad.

Entre otros factores, la racionalidad limitada del votante se reflejaría en los problemas de atención selectiva (Simon 1985:302), la mutabilidad de las preferencias, la tendencia a tomar decisiones que privilegian los beneficios de corto plazo (Przeworski et al., 1999:10) y la prevalencia del juicio intuitivo sobre el razonado (Kahneman, 2002) ${ }^{8}$. Por el contrario, algunos asumen, en general implícitamente, que los funcionarios y expertos poseerían cualidades cognitivas fuertes y que estarían suficientemente informados y preparados para que sus políticas sean tan eficaces y consistentes como ellos pretenden. Se asume también la transparencia y la disponibilidad de información brindada por los funcionarios y los grupos de interés asociados con éstos (Maravall, 1999:193).

¿Cuáles serían las implicancias institucionales, de tipo normativo, del diagnóstico de la racionalidad limitada? En general, se aduce la necesidad de introducir o reforzar filtros múltiples e independientes para corregir las decisiones inconsistentes de los votantes. Para algunos, y en línea con Schumpeter, el filtro puede ser la existencia de una burocracia independiente con poder discrecional (Brennan y Lomasky, 1993: 214217); para otros, los legisladores cumplen el papel correctivo, filtrando y - cuando el caso lo requiere- incluso ignorando las preferencias de los votantes para asegurar el buen gobierno (Stokes, 1999:128). En

\footnotetext{
${ }^{8}$ Pero véase Lupia (2006) para una respuesta a los diagnósticos descriptivos sobre la incompetencia del votante, medida en encuestas y estudios diseñados por elites de expertos en base a presupuestos de un modelo de racionalidad completa.
} 
otras palabras, según la óptica de la racionalidad limitada los representantes deberían actuar (se asume que desean hacerlo) en el mejor interés ciudadano. Afirmar lo anterior no excluiría intentar mejorar la información del electorado, pero siempre dentro de un modelo pensado para que los representantes puedan corregir los problemas cognitivos subyacentes al voto ciudadano.

A partir del presupuesto de racionalidad limitada algunos autores se interesan por mantener una visión política "robusta" (Brennan y Hamlin, 2000:124-125), es decir, orientada a buscar soluciones dentro de las instituciones democráticas. Así, al igual que Buchanan, defienden la descentralización y el federalismo como forma de minimizar los problemas de información para el votante (Brennan y Lomasky, 1993:221), pero a diferencia del economista de Virginia no incluyen en su consideración respuestas extra-políticas — como las provistas por las instituciones de mercado - para proveer los bienes y servicios públicos sobre los cuales se toman gran parte de las decisiones políticas.

Cabe señalar, por último, las teorías que llevan el presupuesto cognitivo bajo análisis a un extremo, y hablan de la "irracionalidad" de la mayoría de las personas, guiadas por errores sistemáticos o por las emociones. Estas teorías provienen, en general, de los economistas. Por citar un ejemplo, para el economista Dan Ariely (2008) la irracionalidad es entendida como un mal cálculo, generalmente basado en circunstancias aleatorias, que suele conducir a la elección de la alternativa más costosa en términos monetarios. El corolario normativo de Ariely es que para evitar el excesivo costo de estas elecciones - especialmente en el campo del cuidado de la salud, de la educación y de los servicios públicos - el gobierno debería regular los mercados, dado que los individuos son irracionales, no aprenden de sus errores, se equivocan sistemáticamente y los mercados sólo pueden funcionar con personas racionales (2008:39-48).

Pero el argumento de la irracionalidad también es utilizado en sentido contrario para criticar las expectativas sobre la eficacia gubernamental. Para Bryan Caplan, ésta fallaría en asegurar un gobierno sensato, pues los políticos suelen atender las demandas de votantes irracionales y prejuiciosos. Las consecuencias de esta dinámica electoral serían perjudiciales para la implementación de políticas públicas eficaces, dada la tendencia de los votantes a subestimar los beneficios del mercado (Caplan, 2007:22,30-39). En consecuencia, este autor propone trasladar 
algunas decisiones al ámbito privado del mercado, y para las decisiones colectivas aconseja implementar un sistema de voto calificado según el grado de alfabetización económica (Caplan 2007:197).

$\mathrm{El}$ argumento de la irracionalidad da por sentado que las falencias cognitivas de los votantes-consumidores son básicamente irreversibles, y que sólo resta mitigar o controlar sus efectos mediante la acción estatal o mediante restricciones al voto. En el caso de Ariely, el supuesto es que los funcionarios poseen cualidades cognitivas suficientes para alcanzar sus propósitos correctivos; en el caso de Caplan, el supuesto es que los economistas se podrán poner de acuerdo acerca de qué decisiones tomar, y que las medidas adoptadas por ellos serán más sensatas en términos de la asignación de recursos públicos. A modo de crítica, en ambos casos se estarían ignorando las falencias cognitivas alternativas de los funcionarios y expertos (Gaus, 2008).

\section{d) Racionalidad deliberativa}

Para los teóricos de la racionalidad limitada el grado de racionalidad de los votantes se presume o se mide a partir de una decisión individual (elegir un candidato o adoptar una medida). A diferencia de ellos, los teóricos de la racionalidad deliberativa hacen hincapié en la superioridad de los mecanismos colectivos de argumentación y justificación para reforzar las cualidades cognitivas en los procesos sociales. Su mirada es fundamentalmente normativa y evaluativa. Así, desde los años 80 Jurgen Habermas desarrolló una teoría de la "racionalidad interpersonal", que es aquella adquirida a través de la práctica de la deliberación colectiva, mediante la cual se invita a los ciudadanos, legisladores y comunicadores a intercambiar argumentaciones justificadas sobre temas y medidas considerados relevantes por todos (Habermas, 1999).

Todas las teorías deliberativas sostienen que mediante la deliberación los agentes políticos pueden superar las limitaciones de su racionalidad individual, y que pueden conocer cuáles son sus intereses y valores. Así, esas teorías comparten con Schumpeter la idea de que la política es un proceso, pero difieren de él en que la política no consiste en competir para ganar votos, sino en transformar las preferencias individuales mediante la discusión racional (Rawls, 1997). En este sentido, 
la deliberación es una instancia necesaria para obtener o legitimar conocimientos que no podrían ser obtenidos o legitimados de otra manera: "la deliberación amplía nuestro conocimiento, revela defectos en el razonamiento, y ayuda a satisfacer la exigencia de atención imparcial de los intereses de todos los afectados" (Nino, 1996:113).

Frente a las teorías que enfatizan la racionalidad mínima del votante a partir de datos empíricos, los teóricos de la deliberación asumen a priori la potencial igualdad en la capacidad que poseen electores y representantes, y la necesidad de desarrollar esa capacidad mediante la justificación mutua de las opiniones políticas. Así, afirman que “...pensamos en las personas como razonables y racionales, como ciudadanos libres e iguales, con los dos poderes morales [capacidad para una concepción de la justicia y capacidad para una concepción del bien]..." (Rawls, 1997:800), o sugieren que el ciudadano se contemple como un "legislador ideal" a la hora de evaluar las leyes y ofrecer una concepción razonable de justicia (Gutman y Thompson, 2000:161).

Sin embargo, a pesar del elevado grado de confianza cognitiva, las prescripciones institucionales de algunos teóricos de la deliberación limitan el ejercicio de la racionalidad deliberativa a grupos específicos como los jueces, los funcionarios públicos (legisladores y ejecutivos) y los candidatos a cargos políticos; los ciudadanos están invitados a deliberar en otros foros públicos, pero no en el foro político (Rawls, 1997:767). La práctica deliberativa es incluso prescindible, pues puede ser eliminada si interfiere con la búsqueda de fines sustanciales. En última instancia, la democracia deliberativa sólo exige que la decisión de omitir la deliberación sea hecha pública y por agentes responsables de rendir cuentas (Gutman y Thompson, 2000:177). Probablemente esta conclusión sea inevitable, dadas las premisas cognitivas del modelo deliberativo, lo que implica que en el caso de que esas exigencias no sean satisfechas se recurra a instancias no deliberativas o se introduzcan importantes restricciones al proceso de modo de asegurar el resultado buscado. También se ha propuesto robustecer el filtro judicial para limitar o minimizar los efectos de una deliberación equivocada que afecte o pueda afectar los derechos individuales (Nino, 1996:202, 204, 207).

Puede pensarse que admitir la necesidad de filtros legislativos, judiciales y deliberativos implica reconocer que el producto de la deliberación pueda no ser el resultado de un mejor conocimiento colectivo. Ello implicaría desconocer que los mismos representantes y jueces a 
veces se comportan como "ignorantes racionales", evitando obtener información correcta a la hora de evaluar y adoptar medidas, o simplificando la complejidad de los temas en aras de maximizar sus objetivos políticos o personales (Rizzo y Whitman, 2008:30-31).

Esta última crítica contrasta con la idea de que no existiría ninguna tensión entre los ciudadanos que deliberan para elegir los objetivos de la asociación política, por un lado, y los legisladores que deliberan para elegir los medios para alcanzar esos objetivos, por el otro (Christiano, 1996:233). Esta postura se acerca más a la confianza schumpeteriana de que existen líderes con cualidades cognitivas (y morales) suficientemente elevadas, y comparte la mirada de los teóricos de la racionalidad limitada respecto de los funcionarios y técnicos, en el supuesto de que éstos pueden conocer cuáles son los intereses específicos de los ciudadanos independientemente de las preferencias de ellos.

\section{e) Ignorancia inevitable}

A partir del aporte de Hayek, las concepciones enfocadas en el hecho de la ignorancia han sostenido que todos los individuos son considerablemente ignorantes de los numerosos factores necesarios para alcanzar sus objetivos. En su opinión, todos somos ignorantes fundamentalmente debido a la complejidad de la realidad social y a la naturaleza limitada del conocimiento humano, la mayor parte del cual no es explícito sino tácito, y se encuentra disperso entre todos los individuos (Hayek, 1978:29-30).

A diferencia de los otros debates que asumen la racionalidad de los gobernantes y no la de los votantes, aclara este autor, desde esta óptica el problema de la ignorancia alcanzaría a gobernantes, ciudadanos, expertos, intelectuales y científicos por igual. En otras palabras, la ignorancia es constitutiva del ser humano; por ende, es inevitable y resistente a nuestros esfuerzos por sortearla. En particular, es fútil - peor aún, puede ser dañino- el intento de los funcionarios y expertos que, inspirados por la "pretensión del conocimiento", creen posible poseer el conocimiento y el poder suficientes para "moldear los procesos sociales a nuestro gusto, conocimiento que de hecho no poseemos" (Hayek, 1974). En contraste con esta pretensión, Hayek postula una "lección de humildad": aceptar que la civilización es producto del esfuerzo libre de 
millones de individuos y no del diseño de una mente (Hayek, 1974). Ese esfuerzo se inscribe en un proceso evolutivo, a partir del cual se produce un aprendizaje de las cosas que salieron mal, es decir, un aprendizaje a partir del fracaso (Hayek, 1978:36).

Las implicancias institucionales de la visión hayekiana son varias. Aceptar la ignorancia inevitable implica, por ejemplo, desplazar la exigencia de obtener el mejor conocimiento posible hacia la necesidad de adoptar procesos e instituciones adecuadas para poder hacer un mejor uso del conocimiento escaso y disperso (Hayek, 1978:26-27). Desde un punto de vista normativo, ello exige adoptar instituciones que nos permitan aprender a través del ensayo y error y elegir así los cursos de acción adecuados, a partir de un "sistema competitivo", el único que nos puede brindar información sobre cómo coordinar mejor nuestras acciones (Hayek, 1979:177-178). En el campo político, una democracia limitada es el mejor arreglo institucional para hacer frente a las exigencias cognitivas, ya que la libre competencia entre propuestas hace posible la creación y difusión de nuevo conocimiento (Hayek, 1978:109-110,117).

Siguiendo a Hayek, Viktor Vanberg (2006) sostiene que a partir del reconocimiento de nuestra ignorancia es que decidimos adoptar reglas de comportamiento, es decir, reglas morales que el curso de la evolución humana nos ha indicado que son racionalmente óptimas en términos de los beneficios buscados. Desde esta óptica, la conducta guiada por el cumplimiento de esas reglas no requiere un análisis racional de costo-beneficio para coordinar las actividades sociales de modo óptimo. En este sentido, escribe el autor, su teoría del rule-following behavior es más apropiada que la teoría de la elección racional a la hora de analizar las conductas humanas.

Cabe efectuar aquí otras comparaciones entre la visión de la ignorancia inevitable y las demás posturas cognitivas. Al igual que los teóricos de la deliberación, los teóricos de la ignorancia inevitable resaltan la naturaleza intersubjetiva del conocimiento: es en sociedad, cooperando con los demás, donde podemos conocer mejor. Pero mientras para los primeros el problema cognitivo se resuelve mediante la argumentación, para los segundos se resuelve en las interacciones individuales que incluyen otros mecanismos sociales para articular los distintos intereses, sentimientos, valores e ideas de las personas. Así, "los procesos políticos discursivos pueden de hecho reducir el espectro, la 
calidad y la complejidad de los procesos comunicativos" (Pennington, 2003:728). Desde esta óptica, las decisiones basadas exclusivamente en la argumentación racional dejan de lado otras habilidades individuales y pautas culturales difíciles de articular, pero también conducentes a resultados socialmente eficaces (Tebble, 2003:262).

Por otra parte, los teóricos de la ignorancia inevitable se alejan de la visión schumpeteriana según la cual la información política es abundante y en principio posible de ser adquirida, mientras que los primeros niegan que esa adquisición sea abundante y/o disponible. Además, mientras Schumpeter confía en burócratas eficaces, la ignorancia inevitable desconfía de sus capacidades cognitivas y de sus tendencias planificadoras, basadas en la pretensión de conocer más de lo que realmente pueden. Por último, los teóricos de la ignorancia inevitable se distinguen de los "irracionalistas" en que aquéllos (elección racional y racionalidad limitada) creen posible encauzar los impulsos y errores de las personas mediante un proceso de aprendizaje gradual, en pos de un mejor uso del conocimiento, mientras que éstos (ignorancia inevitable) parecen resignarse a que exista tal posibilidad.

Por último, y desde un punto de vista descriptivo y evaluativo, Gerald Gaus advierte que la capacidad cognitiva del público, de los funcionarios y de los expertos debe analizarse tanto en términos absolutos como relativos, y respecto de cuestiones específicas. Este autor sostiene que, comparativamente considerados, los expertos suelen predecir las consecuencias económicas de las medidas políticas mejor que el público, si bien en términos absolutos tampoco lo hacen tan bien. Pero en materia de racionalidad moral, concluye, no hay "expertos" y la gente está capacitada para hacer juicios correctos (Gaus, 2008:17-20).

En la medida en que muchas de las decisiones políticas deben ser analizadas a la luz de su legitimidad, al igual que Schumpeter y Vanberg, Gaus también sitúa el plano cognitivo junto al plano moral a la hora de evaluar la dinámica de los actores y el funcionamiento de las instituciones políticas.

\section{Consecuencias de las teorías sobre la racionalidad para las políticas públicas}

De un tiempo a esta parte, las ideas sobre la racionalidad individual han tenido considerable impacto en el diseño de las políticas públicas. Para ilustrar mejor esta idea analizo a continuación 
una postura que presupone una racionalidad limitada en la mayoría de las personas, y que propone atenuar esas limitaciones mediante correctivos o señales emitidas por los funcionarios de gobierno. Tal es la propuesta de Richard Thaler y Cass Sunstein (2003), quienes hablan de un "paternalismo liberal" para defender la idea de que el Estado induzca a los individuos a actuar y tomar decisiones cuyos beneficios no serían advertidos por ellos mismos. En este sentido, su propuesta se distinguiría del paternalismo a secas en que éste controla los recursos o bienes y los asigna o distribuye según un único criterio, sin posibilidad de opciones por parte del individuo. En cambio el paternalismo liberal guiaría a las personas a tomar decisiones en la dirección que - el gobierno estima - es racionalmente óptima respecto de un objetivo dado. Los ejemplos que mencionan en su propuesta van desde obligar a las personas a contratar un seguro automotor y a ahorrar para la vejez, hasta la "invitación" a no fumar y comer comida saludable mediante la adopción obligatoria del menú light en los restaurantes.

El enfoque cognitivo del "paternalismo liberal" se asienta en dos argumentos del tipo de racionalidad limitada: a) un presupuesto empírico según el cual las personas carecen de preferencias definidas, estables $\mathrm{u}$ ordenadas, por lo que sus elecciones dependen del contexto y la presentación de las opciones (2003:1161), y b) un presupuesto normativo que defiende la intervención estatal mínima, es decir, la adopción de requisitos y regulaciones que influenciarían la conducta de las personas, orientándolos “en direcciones sensatas" (2003:1188-1193)9.

El paternalismo liberal ha sido criticado por Mario Rizzo y Donald Whitman, para quienes la noción ignora los clásicos problemas asociados con la regulación estatal de las actividades privadas y con la expansión incesante del radio de acción estatal hacia nuevas órbitas. En este sentido, el paternalismo tendría el potencial de acarrear consecuencias no intencionadas que frustren sus objetivos e impliquen

${ }^{9}$ Thaler y Sunstein alientan a los planificadores estatales a estar más informados acerca de cómo instrumentar sus ideas, llegando a sugerirles la posibilidad de efectuar una deducción estatal automática para fomentar las donaciones de caridad (2003:1193 n.132). En este esquema las donaciones se distinguirían de los impuestos en que permitirían a los individuos "salirse" de esas deducciones si así lo indicaran. Para los autores, los esquemas de opt out se basan en la evidencia de que debido al esfuerzo asociado con la toma de decisiones, los individuos tienden a demorar esas decisiones, por lo que en el ejemplo planteado, un sistema de opt out aseguraría más donaciones que uno de opt in. 
nuevas intervenciones estatales (Rizzo y Whitman, 2008:23,56). Por otro lado, esgrimen estos autores, las falencias cognitivas atribuidas a los individuos también se aplican a los planificadores, quienes tienden a concentrar su mira en el corto plazo (ya que probablemente no estén en sus cargos a la hora de enfrentar los costos o beneficios de sus decisiones como funcionarios).

En la misma línea argumentativa que enfatiza falencias cognitivas para ciudadanos y expertos por igual, Tasic emplea la expresión "ilusión de la profundidad explicativa" para aludir a un exceso de confianza en nuestras capacidades cognitivas. Así — escribe- creemos que "entendemos las causas, los efectos y el funcionamiento interno de mecanismos complejos, eventos y procesos mucho mejor de lo que realmente lo hacemos" (Tasic, 2009:426). De acuerdo con este autor, basado en un amplio repertorio de descripciones de casos puntuales, en el complejo campo de la política los economistas y funcionarios toman decisiones para regular (u omitir regular) las actividades económicas, sin advertir que existen consecuencias secundarias que pueden frustrar sus planes. También suelen incurrir en simplificaciones extremas que luego impiden anticipar la verdadera magnitud de los problemas económicos y sociales que se derivan de esas medidas. Los agentes reguladores y los expertos no sólo ignoran estos factores, sino que además “ignoran su propia ignorancia” (Tasic, 2009:430).

Las críticas al paternalismo liberal y a la ilusión de la profundidad explicativa se inscriben en lo que aquí denominamos la teoría de la ignorancia inevitable, según la cual todos los individuos, incluyendo expertos y funcionarios, presentan sesgos cognitivos a la hora de recabar información y evaluar las consecuencias de las acciones y regulaciones adoptadas a partir del uso de ese conocimiento limitado. En el plano normativo, y en el caso de los funcionarios públicos, entre los instrumentos institucionales para contrarrestar los defectos cognitivos se ha sugerido incluir restricciones procesales y sustantivas a la acción estatal (Rizzo y Whitman, 2008:43, 59, 60)

\section{Algunas consideraciones sobre los sentimientos en política}

En las teorías analizadas en las secciones anteriores, el análisis de las emociones suele presentarse incorporado o enfrentado a la dinámica de la racionalidad. Así, encontramos autores para quienes las emociones 
complementan y 'abren los ojos' de la razón (Elster, 2008; Nussbaum, 1996); son el 'determinante principal' de muchos juicios y conductas, de modo que las elecciones de las personas se ven más influidas por sus sensaciones e impresiones que por un criterio de utilidad (Kahneman, 2002; Kahneman y Tversky, 1984); pueden dificultar la racionalidad (Schumpeter, 1947:304); "esclavizan" la mente (Buchanan y Tullock 1999:304), son una "mala influencia" (Brennan y Lomasky, 1993:217), y corrompen el pensamiento (Caplan, 2007:152), entre otras ${ }^{10}$.

Sobre la influencia mutua entre los sentimientos y la racionalidad hay un debate extenso y específico del que no me ocuparé en esta sección. Me interesa, en cambio, examinar otros abordajes teóricos sobre los sentimientos, en función de las consecuencias que éstos pueden acarrear para las instituciones y políticas públicas. En particular, me ocuparé de los argumentos sobre el miedo, la solidaridad y la compasión, tal como son presentados para justificar —o criticar - el rol del Estado en paliar situaciones que, según esas visiones, despiertan sentimientos asistenciales. Por ejemplo, para Isaiah Berlin las emociones habrían inspirado ciertas perspectivas "terapéuticas" que observan a la gente en función de miedos y ansiedades que el experto piensa puede corregir a través de la acción estatal (Berlin, 1999:87-88). En este sentido, se ha sostenido que los sentimientos humanitarios o compasivos serían la principal inspiración de quienes defienden un rol estatal activo al servicio del bienestar social (Friedman, 2005).

El argumento del miedo puede rastrearse al influyente psicólogo Eric Fromm, quien en su libro Miedo a la libertad retrató al hombre occidental moderno como un individuo cuyos sentimientos de inseguridad y soledad surgen del capitalismo, un sistema donde se vive bajo la falsa ilusión de libertad (Fromm, 1974:132-138) ${ }^{11}$. Fromm proponía rescatar el esfuerzo creativo individual mediante una economía planificada, en la que "toda la nación domine racionalmente las fuerzas sociales y económicas" y que combinara la "planificación desde arriba" con la "cooperación activa" y la fiscalización desde abajo (ibíd., 299-301). En su propuesta,

${ }^{10}$ Se me ha sugerido sumar otros enfoques que aborden los aspectos emocionales y biológicos de la conducta como, por ejemplo, el de Fowler, Baker, y Dawes (2008) y Lakoff (2008). Como no hay suficiente espacio aquí, deberé dejar el tratamiento de este aspecto del tema para futuros trabajos.

${ }^{11}$ Véase un análisis similar al de Fromm en los otros autores de la Escuela de Frankfort como T. W. Adorno, Max Horkheimer y Herbert Marcuse. 
Fromm reconoció el carácter problemático (aunque para él no contradictorio) de combinar la planificación estatal con la actividad espontánea descentralizada, pero supuso que de todos modos era posible conciliarlas.

Curiosamente, el argumento socialista de Fromm hace eco en el argumento liberal de James Buchanan, quien reconoce en muchos individuos el miedo a la libertad, entendido como una predisposición generalizada que demostraría la aversión a enfrentar las responsabilidades y desafíos individuales, con la consecuente apelación a la intervención estatal para solucionar cuestiones que los individuos consideran difícil o improbable de resolver por sí mismos (Buchanan, 2005). Buchanan sostiene que en el pasado el miedo era contrarrestado por la visión religiosa, pero en el mundo secular moderno el Estado ha reemplazado la función consoladora de la religión ${ }^{12}$. Pero a diferencia de Fromm, para quien la inseguridad emocional proviene de una sensación de impotencia individual frente al sistema capitalista que reduce al individuo a una dimensión laboral-instrumental, para el autor norteamericano el miedo a la libertad provendría de la incertidumbre que trae aparejada la libertad, al ampliarse la esfera de oportunidades individuales y consecuentemente multiplicarse la posibilidad de incurrir en decisiones erradas o riesgosas, ocasionando resultados adversos a los fines buscados.

Una preocupación similar a la de Buchanan late también en el análisis de Hayek sobre la "seguridad económica" y sus implicancias para la vida institucional y la libertad (Hayek, 1972: IX; 1978:257 ss.). Esta noción de seguridad se refiere al deseo de las personas de buscar refugio en la posesión de recursos materiales para hacer frente a la incertidumbre y al riesgo (1978:286). Nótese que Hayek no utiliza un lenguaje jurídico — reclamar un derecho a ciertos bienes o exigir el deber de proveerlos - sino más bien uno psicológico: satisfacer el sentimiento de seguridad. El actor individual que tiene en mente no es un ser racional que calcula cómo maximizar su riqueza, sino alguien "perezoso, imprudente y despilfarrador" (1978:61), que pareciera contentarse con un mínimo de seguridad material.

Frente a este actor así retratado, ¿cuáles políticas públicas recomendar? Hayek distingue dos clases de actitudes por parte de funcio-

${ }^{12}$ En este sentido, su opinión sobre el "miedo" prevalente es coherente con su visión de la conducta "litúrgica" de los votantes: ambos estarían facilitados o inducidos por un marco institucional (la debilidad de las instituciones religiosas, acompañada de una fuerte institución estatal, en el primer caso, y las instituciones representativas a gran escala, en el segundo). 
narios y expertos para calmar el sentimiento de inseguridad: la primera busca asegurar una provisión mínima de bienes básicos para todos, mientras que la segunda consiste en determinar resultados finales en función de criterios políticos, controlando o modificando los resultados obtenidos en el mercado. En su opinión el primer camino no lesiona libertades, y si bien se presta a distorsiones y abusos, en principio puede sostenerse, mientras que el segundo camino afecta y restringe las libertades individuales y por ende no es admisible (Hayek, 1972: IX).

La recomendación de Hayek va dirigida a gobernantes "cortos de vista, ansiosos o impacientes" que, movidos por esos sentimientos y por la pretensión de conocer, implementan soluciones erradas e inadmisibles (1978:260-266). En este sentido, el autor austríaco parece respaldar la idea de que la fe en el Estado benefactor y la crítica al capitalismo estarían inspiradas tanto en impulsos sentimentales como en pretensiones planificadoras (pace Friedman, 2005:40).

Una ilustración reciente de la fuerza de los impulsos sentimentales en política se encuentra en la teoría de Ernesto Laclau. Siguiendo los conceptos de Freud y Lacan sobre los lazos libidinales que operan en los grupos sociales, Laclau centra su análisis en los sentimientos que subyacen a las formas de la organización populista. Según este autor, la "razón populista" — que él defiende - sería "ininteligible sin el componente afectivo" (2009:143). Cabe advertir que este componente es doble, ya que incluye un aspecto vertical - los sentimientos de identificación a partir del amor del pueblo por su líder- y un aspecto horizontal: los sentimientos de solidaridad entre las demandas insatisfechas que se agrupan en el concepto de pueblo (2009:81,153).

A diferencia de las lecturas marxistas que restringen la solidaridad a la clase proletaria, para Laclau cualquier demanda democrática puede erigirse en parte solidaria. El éxito del proyecto populista será entonces articular todas esas demandas en un proyecto "hegemónico" y oponerlas a cualquier "otro antagónico". Por un lado, entonces, tenemos lazos afectivos que unen al pueblo, y por el otro, un antagonismo que lo desune de cualquier fuerza opositora. En ambos planos priman los afectos, y hasta podría inferirse que el amor por el líder se nutre del odio a la oposición. Independientemente de las críticas a la "razón populista"13

${ }^{13}$ Para un análisis más detallado del pensamiento de Laclau y algunas críticas véase Salinas (2010). 
he querido señalar con este ejemplo la presencia de los sentimientos en una teoría política de gran difusión actual.

Dentro de los otros aportes teóricos en torno a los sentimientos en política, la filósofa Martha Nussbaum (2003:401-454) se preocupó por analizar el sentimiento de la compasión, que nos llevaría a buscar modos políticos de mejorar la suerte de quienes sufren un dolor inmerecido o desproporcionado. A diferencia de la lectura psicoanalítica de Laclau, quien limita la solidaridad sólo al pueblo, para la filósofa norteamericana una sociedad compasiva siente la necesidad de ayudar a todos los que están peor para así proteger su dignidad humana (ibídem, 414). Contra la sospecha de que las emociones nublan la racionalidad, esta sensibilidad compasiva, la capacidad de "sentir con" los otros, para ella operaría en conjunción con, y no enfrentada a la racionalidad instrumental y a la racionalidad deliberativa (ibídem, 441;454).

En su invitación a ser más compasivos, Nussbaum sugiere promover las emociones compasivas, por ejemplo mediante una educación pública que cultive las artes y humanidades para formar ciudadanos sensibles (ibídem, 425-433). Más importante aún para esta autora es cultivar la imaginación literaria y humanista en los líderes y en quienes toman las decisiones _ como es el caso de algunos jueces - para que puedan discernir mejor las variadas formas de trato desigual a las personas. Una democracia asentada en una noción formal de igualdad sin un sentimiento correlativo, sostiene la autora, es demasiado frágil (ibídem, 442-443).

En un escrito anterior Nussbaum planteaba una forma de complementar el sentimiento de compasión con un sistema de bienestar solventado con impuestos, si bien presentaba el radio de acción de ese sistema como una cuestión de eficiencia librada al balance racional de ensayo y error (1996:51-56). Al margen del juicio sobre las bondades del Estado de bienestar, en la medida en que Nussbaum defiende un estándar de eficiencia para el mismo parece lógico inferir de sus argumentos que la ayuda estatal debe ser justificada en función de resultados evaluados racionalmente, y no sólo porque sentimos que sea buena o apropiada. ¿Quizás este corolario pueda servir de inspiración a los hacedores de políticas públicas que deseen conciliar eficiencia con compasión? 


\section{Conclusiones}

En este trabajo me propuse resumir, clasificar y comparar teorías de diversos autores y disciplinas sobre las disposiciones psicológicas de los actores políticos, sus implicancias institucionales y su impacto en las políticas públicas, con el fin de introducir al lector a las principales posturas sobre el tema.

En el plano cognitivo, apunté dos nociones "fundacionales" -ignorancia inducida y elección racional - que parten de premisas opuestas: la primera detecta una incapacidad cognitiva del votante medio en los sistemas representativos, mientras la segunda lo cree racionalmente capaz, pero indiferente a obtener información política. Ambas se preocupan por los efectos políticos negativos derivados de este paisaje cognitivo, al igual que los teóricos de la racionalidad limitada, para quienes los funcionarios o expertos podrían corregir esos efectos.

En contraste, los "deliberativos" asumen a priori la capacidad racional de todos los actores para deliberar sobre los asuntos públicos y proponen un modelo normativo basado en esta capacidad. Por otra parte, la fe en la deliberación y en la representación es cuestionada por quienes mantienen el supuesto cognitivo más débil, el de la "ignorancia inevitable", y lo aplican a votantes, funcionarios y expertos por igual para alertarlos, por ejemplo, respecto de las consecuencias no intencionadas de las acciones humanas.

En el plano de los sentimientos, examiné algunos argumentos presentados para justificar o criticar el rol del Estado en atender aquellas demandas que, según las visiones respectivas, se basan en los sentimientos del miedo, la solidaridad o la compasión, y que buscarían atenuarlos mediante el asistencialismo, tanto en su versión más clásica del sistema de bienestar social como en su más moderna versión populista.

Si bien las implicancias institucionales de los presupuestos cognitivos y emocionales aquí mencionados son varias, identifiqué, por un lado, las que defienden los mercados y la descentralización política como una forma de minimizar los efectos de los problemas cognitivos de todos los actores políticos, y por el otro las que confían en la capacidad de los políticos y/o expertos tanto para promover los sentimientos de compasión como para introducir mayor racionalidad mediante regulaciones a las decisiones individuales. En relación con este último punto, resumí la visión del paternalismo liberal y de sus críticos para ilustrar algunas de las consecuencias concretas de los presupuestos psicológicos en el campo de las políticas públicas. 


\section{REFERENCIAS}

Ariely, Dan (2008). Predictably Irrational: The Hidden Forces that Shape Our Decisions. New York: Harper Collins.

Berlin, Isaiah (1983) [1964]. "De la esperanza y el miedo liberado". En I. Berlin, Conceptos y categorías. México: Fondo de Cultura Económica. - (1999) [1949]. "Political Ideas in the 20 ${ }^{\text {th }}$ Century". En I. Berlin, Liberty. Henry Hardy (comp.). Oxford: Oxford University Press.

Boudon, Raymond (2003). "Beyond Rational Choice". Annual Review of Sociology, Vol. 29, 1-21.

Brennan, G. y James M. Buchanan (2000) [1984]. "Voter Choice: Evaluating Political Alternatives". En Politics as Public Choice. Indianapolis: Liberty Fund.

Brennan, G. y Alan Hamlin (2000). Democratic Devices and Desires. Cambridge, MA: Cambridge University Press.

Brennan, G. y Loren Lomasky (1993). Democracy \& Decision: The Pure Theory of Electoral Preference. Cambridge, MA: Cambridge University Press.

Buchanan, James M. (2005). “Afraid to Be Free: Dependency as Desideratum”. Public Choice 124: 19-31.

Buchanan, James M. y Gordon Tullock (1999) [1962]. The Calculus of Consent: Logical Foundations of Constitutional Democracy. Indianapolis: Liberty Fund.

Buchanan, James M. y Richard E. Wagner (2000) [1977]. Democracy in Deficit: The Political Legacy of Lord Keynes. Indianapolis: Liberty Fund.

Caplan, Bryan (2007). The Myth of the Rational Voter: Why Democracies Choose Bad Policies. Princeton: Princeton University Press.

Christiano, Thomas (1996). The Rule of the Many: Fundamental Issues in Democratic Theory. Boulder and Oxford: Westview Press.

Converse, Philip E. (1964). "The Nature of Belief Systems in Mass Publics". En David E. Apter (ed.), Ideology and Its Discontents. New York: The Free Press of Glencoe.

Damasio, Antonio (2005) [1994]. Descartes'Error: Emotion, Reason and the Human Brain. USA: Penguin Books.

Downs, Anthony (1957). An Economic Theory of Democracy. New York: Harper \& Row.

Elster, Jon (2008). Reason and Rationality. Princeton: Princeton University Press.

Fowler, James H., Laura A. Baker y Christopher T. Dawes (2008). "Genetic Variation in Political Participation". American Political Science Review, Vol. 102, No. 2, pp. 233-248.

Frank, Robert H. (1988). Passions Within Reason. The Strategic Role of Emotions. New York \& London: W. W. Norton \& Company.

Friedman, Jeffrey (2005). "Popper, Weber and Hayek: The Epistemology and Politics of Ignorance". Critical Review, 17, 1-18.

Fromm, Erich (1974) [1941]. Miedo a la libertad. Buenos Aires: Amorrortu. 
Gaus, Gerald (2008). "Is the Public Incompetent? Compared to Whom? About What?". Critical Review: A Journal of Politics and Society, 20 (3), 291-311.

Gluck, Andrew L. (2007). Damasio's Error and Descartes'Truth: An Inquiry into Epistemology, Metaphysics and Consciousness. Scranton \& London: University of Scranton Press.

Gutmann, Amy y Dennis Thompson (2000). "Why Deliberative Democracy Is Different". Social Philosophy and Policy, Vol. 17:1, pp. 161-180.

Habermas, Jürgen (1999) [1996]. La inclusión del otro: estudios de teoría política. España: Paidós.

Hardin, Russell (2000). "Democratic Epistemology and Accountability". Social Philosophy and Policy, 17 (1), 110-126.

Hayek, Friedrich A. (1972) [1944]. The Road to Serfdom. Chicago: University of Chicago Press.

(1974). "La pretensión del conocimiento". En Los Premios Nobel de Economía 1969-1977. México: Fondo de Cultura Económica. (1978) [1960]. The Constitution of Liberty, Chicago: The University of Chicago Press.

(1979) [(1952]. The Counter-Revolution of Science. Studies on the Abuse of Reason, Indianapolis: Liberty Press.

Hobbes, Thomas (1651). Leviathan, en http://www.gutenberg.org/ebooks/3207.

Kahneman, Daniel (2002). "Maps of Bounded Rationality: A Perspective on Intuitive Judgement and Choice". Discurso de Aceptación del Premio Nobel de Economía.

Kahneman, Daniel y Amos Tversky (1984). "Choices, Values and Frames". American Psychologist, abril, 342-350.

Kant, Immanuel (1784). “What is Enlightenment?”. En http://www.fordham. $\mathrm{edu} / \mathrm{halsall} / \mathrm{mod} / \mathrm{kant}$-whatis.asp.

Laclau, Ernesto (2009) [2005]. La razón populista. Buenos Aires: Fondo de Cultura Económica.

Lakoff, George (2008). The Political Mind: Why You Can't Understand 21stCentury American Politics with an 18th-Century Brain. Viking Adult/ Penguin.

Locke, John (1954) [1690]. The Second Treatise of Government. Thomas Peardon, editor. New York: The Liberal Arts Press.

Lupia, Arthur (2006). "How Elitism Undermines the Study of Voter Competence". Critical Review, 18 (1-3), 217-232.

Macedo, Stephen (comp.) (1999). Deliberative Politics: Essays on Democracy and Disagreement. Oxford: Oxford University Press.

Maravall, José M. (1999). “Accountability and Manipulation”. En A. Przeworski, S. Stokes y B. Manin (comp.), Democracy, Accountability and Representation. Cambridge: Cambridge University Press.

Nino, Carlos S. (1996). The Constitution of Deliberative Democracy. New Haven: Yale University Press.

North, Douglass C. (1990). “A Transaction Cost Theory of Politics". Journal of Theoretical Politics, 2, 355-367. 
Nozick, Robert (1995) [1993]. La naturaleza de la racionalidad. Barcelona y Buenos Aires: Ediciones Paidós.

Nussbaum, Martha (1996). "Compassion: The Basic Social Emotion". Social Philosophy and Policy, 13, 57-78.

(2003) [2001]. Upheavals of Thought: The Intelligence of Emotions, Cambridge, MA: Cambridge University Press.

Olson, Mancur (1971) [1965]. The Logic of Collective Action: Public Goods and the Theory of Groups. Cambridge: Harvard University Press.

Pennington, Mark (2003). "Hayekian Political Economy and the Limits of Deliberative Democracy”. Political Studies, 51, 722-739.

Pincione, Guido y Fernando Tesón (2006). Rational Choice and Democratic Deliberation: A Theory of Discourse Failure. Cambridge: Cambridge University Press.

Prisching, Manfred (1995). “The Limited Rationality of Democracy: Schumpeter as the Founder of Irrational Choice Theory". Critical Review, 9 (3), 301-324.

Przeworski, A., S. Stokes y B. Manin (comp.) (1999). Democracy, Accountability and Representation. Cambridge: Cambridge University Press.

Rawls, John (1997). "The Idea of Public Reason Revisited". University of Chicago Law Review, 64 (3), 765-807.

Rizzo, Mario J. y Douglas Whitman (2008). "Little Brother is Watching You: New Paternalism on the Slippery Slopes". NYU Law School, Public Law Research Paper No.08-12.

Salinas, Alejandra (2011). "Populismo, democracia, capitalismo: La teoría política de Ernesto Laclau". Crítica. Revista de Teoría Política Contemporánea, Año 1, No 1, pp. 168-188. Disponible en http://www. fcs.edu.uy/archivos/REvista\%20completa.pdf.

Schumpeter, Joseph (1947) [1942]. Capitalismo, Socialismo y Democracia. Buenos Aires: Editorial Claridad.

Simon, Herbert A. (1985). "Human Nature in Politics: The Dialogue of Psychology with Political Science". APSR, 79, 293-304.

Smith, Adam (1982) [1759-1790]. The Theory of Moral Sentiments. Indianapolis: Liberty Fund [reimpresión de la edición de Oxford University Press de 1976-1979].

Somin, Ilya (1998). "Voter Ignorance and the Democratic Ideal". Critical Review 12 (4), 413-458.

Stokes, Susan (1999). "What Do Policy Switches Tell Us About Democracy?". En A. Przeworski, S. Stokes y B. Manin (comp.) (1999). Democracy, Accountability and Representation. Cambridge: Cambridge University Press.

Tasic, Slavisa (2009). "The Illusion of Regulatory Competence". Critical Review, 21(4), 423-436.

Tebble, Adam (2003). “Does Inclusion Require Democracy?”. Political Studies, 51, 197-214.

Thaler, Richard H. y Cass R. Sunstein (2003). "Libertarian Paternalism is Not an Oxymoron". The University of Chicago Law Review, 70 (4), 11591202. 
Tocqueville, Alexis de (2000) [1835]. La democracia en América. México: Fondo de Cultura Económica.

Vanberg, Viktor J. (2006). "Rationality, Rule-Following and Emotions: On the Economics of Moral Preferences". Max Planck Institute.

Weyland, Kurt (2006). Bounded Rationality and Policy Diffusion: Social Sector Reform in Latin America. Princeton, NJ: Princeton University Press.

Wohlgemuth, Michael (2007). "Schumpeterian Political Economy and Downsian Public Choice: Alternative Economic Theories of Democracy". Walter Eucken Institut, Discussion Paper 02/7. 